\title{
Incorporación de la nutrición a las iniciativas de la calidad en cirugía electiva
}

\section{Incorporating nutrition into quality improvement initiatives in elective surgery}

Incorporando nutrição às iniciativas de melhoria da qualidade em cirugia eletiva

Juan B. Ochoa Gautier*

https://doi.org/10.35454/rncm.v3n1.025

Se estima que cada año se realizan más de 300 millones de cirugías en todo el mundo. Se desconoce la cantidad de complicaciones que ocurren después de la operación, pero puede ser de $10 \%$ en tipos específicos de cirugía. En la cirugía colorrectal, las complicaciones quirúrgicas pueden ser hasta de $30 \%$. Las complicaciones quirúrgicas aumentan significativamente el costo de la atención y gravan los recursos del sistema de salud ${ }^{(1)}$.

Múltiples ensayos clínicos cuidadosamente realizados han demostrado que las intervenciones específicas pueden prevenir o mejorar la incidencia de complicaciones. Estos se denominan estudios de "eficacia", ya que están diseñados para determinar una causa y efecto entre una intervención (por ejemplo, profilaxis antibiótica) y un efecto (idealmente beneficioso) sobre los resultados clínicos. Así, por ejemplo, el uso de un antibiótico justo antes de comenzar la cirugía (causa) se asocia con una disminución en el riesgo de infecciones (efecto). Cuanto mejor es la calidad del estudio, más fuerte se determina la evidencia de eficacia. Los estudios de alta calidad son generalmente prospectivos, aleatorios y doble ciego y la evidencia generada a partir de este tipo de estudios se llama evidencia de nivel 1 de beneficio clínico.

La práctica clínica debe incorporar intervenciones donde se haya determinado la eficacia (un beneficio clínico) mientras se abandonan las prácticas donde no se han observado beneficios o incluso daños. Esto se llama atención basada en evidencia. Sin embargo, esto se hace de manera inconsistente. Como resultado, las

\footnotetext{
* juan.ochoa@ochsner.org
}

complicaciones quirúrgicas continúan ocurriendo. Por ejemplo, el uso inconsistente del lavado de manos antes de ingresar a la habitación del paciente se asocia con un aumento persistente del riesgo de infección a pesar de la evidencia clara de que el lavado de manos disminuye drásticamente el riesgo de infección ${ }^{(2)}$.

A pesar de la clara evidencia de eficacia, muchas intervenciones, en particular aquellas que parecen ser simples, no se incorporan rutinariamente en la práctica clínica diaria. De hecho, a pesar de la obvia motivación altruista de lograr que los pacientes alcancen los mejores resultados, existen barreras importantes para implementar iniciativas basadas en evidencia que apunten a mejorar la atención.

Las iniciativas destinadas a ayudar a los médicos a incorporar la atención basada en la evidencia en sus prácticas se denominan iniciativas de mejora de la calidad (QI). La incorporación de iniciativas dirigidas a mejorar la calidad de un producto se aplicó por primera vez en las líneas de ensamblaje y en la industria de las aerolíneas. Estas proporcionan un mecanismo estructurado de cambio de práctica.

El valor se define como una fórmula matemática con resultados clínicos en el numerador y el costo de la atención en el denominador. El valor de QI se determina por su efecto sobre los resultados clínicos o el costo o ambos. Dado que los malos resultados clínicos están asociados a un mayor costo, es lógico esperar que las iniciativas de QI puedan aumentar el valor al mejorar los resultados clínicos y al mismo tiempo disminuir el costo.

El QI en cirugía se considera una iniciativa esencial de salud pública. En parte, dado que la información sobre los resultados ahora se presentan en forma instantánea al público en general, la mala calidad de la atención se con- 
sidera inaceptable. En otras palabras, los valores sociales han cambiado drásticamente desde la idea del destino ("usted -el paciente-, tuvo mala suerte de que haya desarrollado una infección postoperatoria") al concepto de responsabilidad ("usted -el cirujano-, fue responsable del desarrollo de un infección postoperatoria debido a la mala calidad de la atención”). Los resultados quirúrgicos y la incidencia de complicaciones quirúrgicas de un hospital determinado o incluso de un cirujano específico, se hacen cada vez más públicos.

Las intervenciones nutricionales como parte de las iniciativas de QI en cirugía se pueden resumir en 4 categorías: a) diagnóstico de desnutrición, b) intervención nutricional preoperatoria, c) evitar el ayuno preoperatorio, $\mathrm{yd}$ ) ingesta oral temprana postoperatoria (Tabla 1). A continuación se describe de manera detallada la revisión de estas diferentes intervenciones:

Tabla 1. Las iniciativas de mejora de la calidad destinadas a mejorar los resultados en pacientes sometidos a cirugía se dividen en las siguientes categorías:

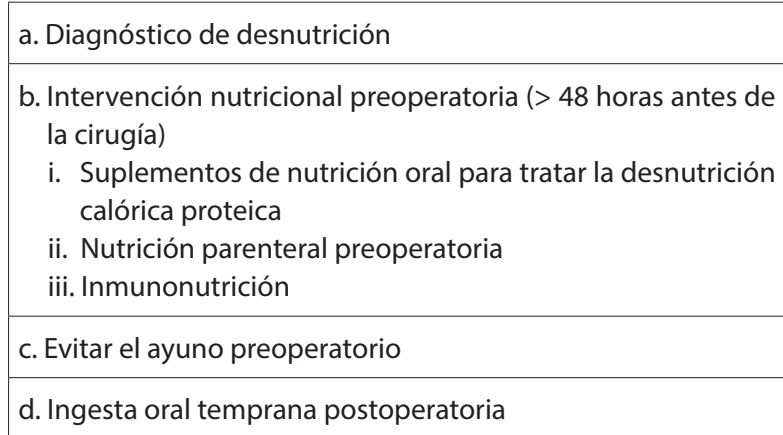

\section{DIAGNÓSTICO DE DESNUTRICIÓN}

La desnutrición es causada por muchos factores. Independiente de estos factores, la desnutrición es una causa importante de malos resultados clínicos y un mayor costo. Hay varias formas de desnutrición y múltiples clasificaciones diferentes. Comúnmente, la desnutrición es causada por la falta de ingesta de alimentos. Sin embargo, es difícil desnutrir a un individuo sano si hay acceso a un suministro de alimentos de calidad. La ingesta adecuada de alimentos y las intervenciones de nutrición médica están disponibles para la mayoría de los pacientes. A pesar de esto, la desnutrición es terriblemente frecuente en los pacientes hospitalizados en todo el mundo y puede observarse en $30 \%$ o más de las personas con enfermedades agudas. Desafortunadamente, la progresión a la desnutrición es implacable en presencia de inflamación aguda o crónica y en muchas enfermedades. Esto ocurre a pesar de la terapia de nutrición óptima. Por lo tanto, si bien diagnosticar la presencia de desnutrición, en particular antes de la cirugía, es esencial como determinante del pronóstico en el riesgo de complicaciones, nuestra capacidad para intervenir con éxito aún es limitada. Hay mucho más por aprender ${ }^{(3)}$.

Un problema importante que complica aún más nuestra comprensión de la desnutrición es la presencia de obesidad. Vista por primera vez en los países del primer mundo en la década de 1970, particularmente en los Estados Unidos, la obesidad es ahora una pandemia que ha llegado a la mayoría de los países. Antes de esta pandemia, los pacientes que estaban enfermos desarrollaban desnutrición proteica y calórica. Las intervenciones nutricionales tradicionales estaban destinadas a prevenir la acumulación de un déficit calórico administrando cantidades modestas de proteínas. En pacientes obesos con enfermedades agudas / graves, no existe un beneficio claro el intentar llegar a metas calóricas. Por el contrario, se observa un beneficio en estos pacientes cuando se incrementa el suministro de proteína y al mismo tiempo se disminuye la cantidad de calorías no proteicas administradas. Este enfoque se conoce como dietas hipocalóricas hiperproteícas. Comienzan a aparecer nuevas terapias destinadas a diagnosticar y tratar con éxito la desnutrición proteica que además permiten movilizar los depósitos energéticos grasos en el paciente obeso ${ }^{(4,5)}$.

\section{INTERVENCIONES PREOPERATORIAS (> 48 HORAS ANTES DE LA CIRUGÍA)}

Los pacientes que están programados para una cirugía pueden darse el lujo de tener el tiempo suficiente para realizar con éxito una intervención nutricional dirigida a reducir el riesgo de complicaciones para mejorar los resultados quirúrgicos. Las intervenciones preoperatorias en esta categoría se dividen en dos grupos distintos, que incluyen nutrición parenteral preoperatoria e inmunonutrición. El uso de nutrición parenteral preoperatoria siete días antes de la cirugía ha demostrado un beneficio claro y objetivo en pacientes prospectivos aleatorizados que reducen las complicaciones en el paciente con desnutrición proteico-calórica severa. Paradójicamente, la nutrición parenteral preoperatoria puede aumentar el riesgo de infecciones postoperatorias utilizadas en pacientes que no están desnutridos o desnutridos de leve a moderada. La nutrición parenteral preoperatoria es costosa y difícil de implementar. Por lo tanto, debe usarse de forma selectiva y bajo la supervisión de un especialista en nutrición. 
Inmunonutrición es el nombre dado a una serie de fórmulas que complementan aminoácidos específicos (en general arginina o glutamina), ácidos grasos omega-3 y con frecuencia nucleótidos, los cuales se agregan a una fórmula nutricional completa estándar. La inmunonutrición a base de arginina (ABIN, por su sigla en inglés) ha sido una de las intervenciones nutricionales más rigurosamente estudiadas en diferentes poblaciones de pacientes ${ }^{(6)}$. Cuando se usa antes de la cirugía electiva ( 5 a 7 días) y después de la cirugía ( 5 a 10 días), ABIN consistentemente produce una disminución de las complicaciones, en particular las complicaciones infecciosas. La inmunonutrición a base de arginina ha sido probada contra los suplementos nutricionales orales estándar (dietas isocalóricas e isoprotéicas) demostrando un beneficio más allá de la suplementación nutricional oral genérica. Se han estudiado múltiples poblaciones quirúrgicas, incluidos pacientes con cáncer de cabeza y cuello, aquellos sometidos a cirugía cardíaca, diferentes tipos de cáncer gastrointestinal, incluyendo cáncer de colon, cirugías urológicas y ginecológicas, entre otros. Los resultados no dejan lugar a dudas; ABIN debe ser un estándar de atención en cualquier paciente que se someta a una cirugía electiva mayor donde se espera una alta tasa de complicaciones. Si bien todavía hay lagunas en el conocimiento para poblaciones específicas de cirugía electiva, ningún estudio realizado en pacientes de cirugía electiva ha mostrado daño. Este no es el caso con otras poblaciones de pacientes, como los ingresados en la UCI con shock séptico o daño potencial observado. Además, no se ha observado un beneficio claro (probablemente debido a dificultades logísticas en la administración de $\mathrm{ABIN}$ ) en pacientes quirúrgicos no selectivos, incluido el trauma.

\section{EVITAR EL AYUNO PREOPERATORIO}

Tradicionalmente, los pacientes sometidos a cirugía electiva con frecuencia quedan "sin nada vía oral NPO" durante muchas horas. Sin embargo, el ayuno preoperatorio no es necesario ni aconsejable. En particular, el suministro de pequeñas cantidades de glucosa hasta dos horas antes de la cirugía y durante las primeras 24 horas después de la operación se asocia a una disminución de la resistencia a la insulina y un mejor control de la glucosa. La carga de carbohidratos como se ha llamado a esta práctica, ahora se considera un estándar de atención en la recuperación mejorada después de los protocolos de cirugía.

\section{INGESTA ORAL POSTOPERATORIA PRECOZ}

$\mathrm{Al}$ igual que el ayuno preoperatorio, hay poca evidencia de que el ayuno postoperatorio sea necesario. La atención basada en la evidencia que incorpora protocolos cuidadosos para alentar la ingesta postoperatoria temprana puede acortar la duración de la estancia hospitalaria, la motilidad gastrointestinal y la satisfacción del paciente. La tolerancia gastrointestinal se ve reforzada por otra práctica basada en la evidencia, incluida una disminución en el uso de opioides y el uso cuidadoso de líquidos por vía intravenosa.

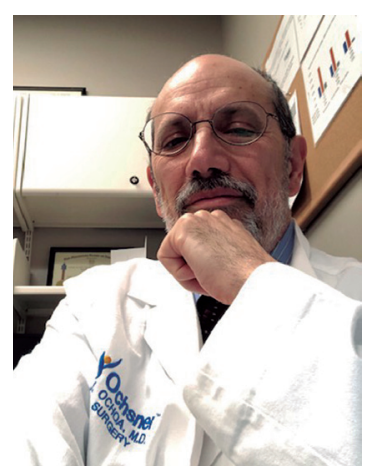

Juan B. Ochoa Gautier, MD, FACS, FCCM se graduó de sus estudios médicos de la Universidad Pontificia Bolivariana y de su primera residencia en cirugía general de la Universidad de Antioquia, ambas en Medellín, Colombia. En 1989 se trasladó a los Estados Unidos en donde realizó su segunda residencia en cirugía general en la Universidad de Pittsburgh y estudios avanzados de posgrado en investigación y en cuidados intensivos. Su carrera académica se ha enfocado a entender las necesidades metabólicas y nutricionales del paciente quirúrgico y el paciente en estado crítico.

\section{Referencias bibliográficas}

1. Weiser TG, Haynes AB, Molina G, et al. Size and distribution of the global volume of surgery in 2012. Bull World Health Organ. 2016;94:201-9F.

2. Corso E, Hind D, Beever D, et al. Enhanced recovery after elective caesarean: a rapid review of clinical protocols, and an umbrella review of systematic reviews. BMC Pregnancy Childbirth. 2017;17:91.

3. Ochoa Gautier JB. Quick Fix for Hospital-Acquired Malnutrition? JPEN J Parenter Enteral Nutr. 2016;40:302-4.

4. Ochoa Gautier JB, Machado FR. Early nutrition in critically ill patients: feed carefully and in moderation. JAMA. 2013;309:2165-6.

5. Ochoa Gautier JB, Martindale RG, Rugeles SJ, et al. How Much and What Type of Protein Should a Critically Ill Patient Receive? Nutr Clin Pract. 2017;32:6S-14S.

6. Drover JW, Dhaliwal R, Weitzel L, Wischmeyer PE, Ochoa JB, Heyland DK. Perioperative use of arginine-supplemented diets: a systematic review of the evidence. J Am Coll Surg. 2011;212:385-99. 